\title{
FORECAST MODELS FOR URBAN DYNAMICS (Kathmandu Valley)
}

\author{
Laxman Basnet \\ Department of Mathematics, Kirtipur. \\ Email Address: me_laxman86@yahoo.com
}

\begin{abstract}
The climatic signature of global warming is both local and global. The forcing by increasing greenhouse gases is global, so there is clearly a global component to the climatic signature. Moreover, the damaging impacts of global warming are manifesting themselves around the world in the form of extreme weather events like storms, tornadoes, floods and droughts, all of which have been escalating in frequency and intensity. Furthermore, it is a well-known fact that there is high degree of uncertainty surrounding projections of basic climate variables, such as temperature and precipitation. However, numerous authors have explored many of these effects individually and have begun exploring the interactions between climate changeinduced impacts in different sectors of urban activities. Therefore, it is safe to say that an attempt to conduct a definitive, comprehensive analysis of all the potential impacts of climate change on the urban structure is premature at present. This communication attempts to examine the trends in maximum monthly urban temperature fluctuations. Analysis reveals increasing trends in urban temperature fluctuations showing effect of Kathmandu industrializations. Forecast models also suggest future scenario with respect to occurrence of extreme temperature. The analysis carried out in this work would be useful for urban planners for sustainable future development, economists and environmentalists etc.
\end{abstract}

Let $\mathrm{C}$ be an urban complex (such as a city with its entire infrastructure) and $\mathrm{U}_{\mathrm{C}}$ the urban system associated with $\mathrm{C}$. We shall consider $\mathrm{U}_{\mathrm{C}}=\mathrm{U}_{\mathrm{C}}\left(\mathrm{P}_{\mathrm{C}}, \mathrm{S}_{\mathrm{C}}, \mathrm{E}_{\mathrm{C}}\right)$ where, $\mathrm{P}_{\mathrm{C}}$ is the set of physical indicators like population, land, population density and infrastructure like developed and undeveloped areas etc. $\mathrm{S}_{\mathrm{C}}$ is the set of socio-economic factors and $\mathrm{E}_{\mathrm{C}}$ is the set of environmental indicators like pollution and climatic variability (land and sea surface temperature, frequency of hazards like cyclones etc.). All these sets are finite. The urban development can refer to both growth and decline. If we represent the growth of an urban complex by $\mathrm{G}_{\mathrm{C}}$, then it will be the rate of increase of $\mathrm{U}_{\mathrm{C}}$ with respect to time so that $\mathrm{G}_{\mathrm{C}}=\frac{d U c}{d t}$. $\mathrm{G}$ is thus a system resulting from the complex dynamical interactions of $\mathrm{P}, \mathrm{S}$, and $\mathrm{E}$ in a certain time interval. Furthermore, $\mathrm{P}, \mathrm{S}$, and $\mathrm{E}$ which are called generator functions or the complexity of the system $U$ involve finite variables depending on time. On occasions these may be random making the situation complex. The urban growth $G$ can be considered a system itself depending on $\mathrm{P}, \mathrm{S}$, and $\mathrm{E}$. Thus, we can consider $\mathrm{G}=\mathrm{G}(\mathrm{P}, \mathrm{S}, \mathrm{E})$. In the following we shall study the urban growth $\mathrm{G}$ in the perspective of the variability in $\mathrm{P}$ and $\mathrm{E}$ only so that $\mathrm{G}=\mathrm{G}(\mathrm{P}, \mathrm{E})$. We shall also study the interactions of $\mathrm{P}$ and $\mathrm{E}$. The generator function $\mathrm{P}$ and $\mathrm{E}$ are themselves subsystems of the system $\mathrm{G}$.

We begin by reviewing the global and local trends of urban growth and then separately study the components $\mathrm{P}$ and $\mathrm{E}$ of urban growth $\mathrm{G}$. The component $\mathrm{P}$ of $\mathrm{G}$ corresponds to the physical growth of the urban complex $\mathrm{C}$ under consideration i.e. the growth of urban population and area. As a consequence of urban growth, a settlement comprising of small population limited to a small area can be gradually transformed into a town, city and megacity. The process usually gets impetus from the massive population movements from rural to urban areas. The urban growth phenomenon associated with a particular urban complex at first seems to be very local but in the perspective of international migrations, globalization of socio-economic activities and environmental issues like global warming, it simultaneously translates into global one. The component $\mathrm{E}$ of $\mathrm{G}$ corresponds to the environmental issues associated with an urban complex $\mathrm{C}$. There are a number of issues requiring attention and those which are particularly related to climatic variability. Climatic variability may be natural or arising from natural causes or may be anthropogenic arising due to non-friendly attitude of humans towards environment. There are various strands of the study of the links between urban growth and climatic variability. But we restrict ourselves to the study of the temperature variations associated with urban mega-complexes. It is interesting to study the impact of these temperature variations on the urban population. In this regard we have given stress to the possibility of increase in the number of tropical cyclones which can create hazards in the coastal populations. This is why we have selected the mega-city of Kathmandu to study as a test case.

The following points are sufficient to gauge the importance of our selection.

a) As regards population and population density Kathmandu is among one of the top of the world

b) It has a very high population growth rate

c) Though it is considered to be a planned city but problems related to its unplanned part which consists of slums seems to overwhelm those of the planned part. 
d) It is considered a city having a moderate climate as compared to the other parts of the country. But now spells of intense heat can be easily observed in the records of past few decades.

e) It is the economic hub of Nepal as it is Kingdom of Nepal.

The situation arising from the very high population growth rate and the increasing number of Kathmandu is clearly hazardous and it will be no exaggeration if we consider high level of population concentration pollution itself. We are of the opinion that the enhanced level of anthropogenic activities is increasing the environmental burden. So we shall stress upon (d) as well and will see that how the extreme temperatures are behaving and whether these extremes are under the influence of human activities or not.

In the perspective of Kathmandu the urban growth $G$ will be a system $G=G(P, S, E)$ with $P, S$ and $E$ as its subsystems where $\mathrm{P}=\mathrm{P}(\mathrm{p}(\mathrm{t}), \mathrm{A}(\mathrm{t}))$ and $\mathrm{E}=\mathrm{E}\left(\mathrm{T}_{1}(\mathrm{t}), \mathrm{T}_{\mathrm{ss}}(\mathrm{t})\right), \mathrm{p}, \mathrm{A}, \mathrm{T}_{1}, \mathrm{~T}_{\mathrm{ss}}$ and $\mathrm{t}$ represent the population, area, land temperature, sea surface temperature and time respectively. The subsystem $P$ can further be considered as $P=P_{s}(p(t), A(t))+P_{\text {us }}(p(t)$, $A(t))$ where $P_{s}$ and $\mathrm{P}_{\text {us }}$ are the settled and unsettled parts respectively of an urban complex. $\mathrm{P}$ and $\mathrm{E}$ and in turn $\mathrm{G}$ can be considered as physical processes. The subsystem $\mathrm{S}$ because of its prime importance has worth for a separate study and will be considered somewhere else.

Key words: urban complex; density models; pivot; global techniques; log-linear transformation

\section{Introduction}

This paper analyses the distribution of some towns of Kathmandu valley. This local analysis refers to the mutual relationship of Kathmandu in a district. In contrast, in case of town wise distribution of Kathmandu the present study performs global analysis which refers to the relationship of a selected valley of a particular town. The global analysis can be performed in two ways. The Direct-Global (DG) method that utilizes the densest valley among all the towns as pivot (the DG pivot) and the ReverseGlobal (RG) method that utilizes the least populated valley among all the towns as pivot (the RG pivot). This study also develops the Distance Gradient Density Model (FDGDM) for the analysis of thepopulation density distribution of Kathmandu. This is an enhancement of the flattened gradient density model(FGDM). The appropriateness of FDGDM is tested with the help of Log-Linear Transformation Model (LLTM) and Log- Exponential Transformation Model (LETM). It is shownthat LETM is more appropriate than LLTM.

We have first developed DG and RG techniques and then the DG and RG analysis is performed. To forecast a better population density distribution of the Kathmandu in the towns FDGDM is developed . The construction of FDGDM involves the distances between densest or least populated of the towns and the respective pivots. The appropriateness of the model is checked with the helpof Log-Linear Transformation Model (LLTM) and Log- Exponential Transformation Model (LETM). Reverse- Global technique appears to be more appropriate than the Direct-Global technique.

\section{Materials and Methods}

The material consists of the population and area data of Kathmandu . The Microsoft excels and ARCVIEW are used to arrange these data and maps. On the basis of these calculations population densities of Kathmandu under consideration is determined. The data such generated analyzed with the help of gradient density models developed with the help of DGT and RGT. A discussion of DGT and RGT and gradient density models is as follows.

\subsection{Direct-Global and Reverse-Global Techniques(DGT \& RGT)}

As said earlier, DG and RG approaches describe the distribution pattern of Ktm with respect to the DG pivot(densest valley) and the RG pivot (least populated valley). 


\subsubsection{Direct-Global technique (DGT)}

Let $T 1 D \ldots T 4 D$ be the densest valley of 4 towns ( Kathmandu, Lalitpur, Bhaktapur and Kirtipur) $1 T$ .. $4 T$ respectively ( $T 1 D>T 2 D>\ldots>T 4 D$ ). The densest valley among $T 1 D \ldots T 4 D$ is the DG pivot represented by $D G P D$ ( $T 1 D$ in our case). DGT is based on calculations with respect to DG pivot. The distances ( $D G P D$, Ti D ), ( Tj D , Tk D),

$i, j, k=1 \ldots 4$ and population densities $d i=\mathrm{pi} / \mathrm{a} i(\mathrm{p} i$ and aiare the population and area of $T i D$ respectively) are determined. (( $D G P D, T i D), d i)$ describe the town wise DG pattern of the distribution of $\mathrm{Ktm}$.

\subsubsection{Reverse-Global Technique (RGT)}

In contrast to DGT, the RGT utilizes the least populated Kathmandu . Among all the dense. Let $T 1 L P$ ... T4LP be the Least Populated towns $1 T \ldots 4 T$ respectively $(T 1 L P>T 2 L P>\ldots>T 4 L P$ ). The Least among $T 1 L P \ldots T 4 L P$ is the RG pivot represented by $R G P L P$ ( $T 1 L P$ in our case). RGT is based on calculations with respect to RG pivot. The distances ( $R G P L P, T i L P),(T j L P, T k L P) i, j, k=1 \ldots 4$ and population densities $d i=\mathrm{p} i / \mathrm{a} i(\mathrm{p} i$ and aiare the population and area of $T i L P$ respectively) are determined.

\subsection{Gradient Density Models}

Gradient Density Models basically forecast the population density distribution with respect to distances betweentheir locations. Such models for the case of district wise distribution pattern of KTM isdeveloped. These models are in fact, Negative Exponential Models (NEM) represented by

$D(x)=D 0$ e- $b x$ to studythe distribution of density these models utilize a gradient coefficient $b$ with respect to a fixed location. FGDM developed in 2007 modifies these models by using various values of $\beta$ to determine the population density.

\subsubsection{Flatten Gradient density model (FGDM)}

The mono-pivotal and multi pivotal Flatten Gradient Density Model (FGDM) is developed. A

mono-pivotal model is applied. We first determine the densest valley of the district under consideration. Then the distances of all the remaining from the densest valleyare calculated. Then considering the densest KA of the district as pivot the population density of a valley isforecasted with the help of the calculated distances and the gradient coefficients $\beta$. The multi-pivotal model appeared to be better than the mono-pivotal model. $D(r)=D 0$ e- $\beta r$

Where $D(r)=$ Population density of valley at a distance $r$

$r=$ Distance square kilometers

$D 0=$ Density at a point

$\mathrm{e}=$ Base of the natural logarithm (approx 2.71828)

$\beta=$ Distance gradient coefficient parameter

\subsubsection{Global Flatten Gradient Density Models(GFGDM)}

In case of the town wise distribution of the city of Kathmandu, Global Flatten Gradient Density Model (GFGDM) is developed for forecasting the population density distribution of the valley using global techniques. GFGDM involvesthe distances between densest or least populated valley of the towns providing better forecasts of the population densities of the valley. The forecasts based on direct global analysis and reverse global analysis utilize the distances calculated with the help of Direct Global Technique and Reverse Global Technique respectively. 


\subsection{Appropriateness of (GFGDM)}

In this section we will test the appropriateness of the reverse and direct methods by using LogLinearTransformation Model (LLTM) and Log-Exponential Transformation Model (LETM). The former one is linearin nature whereas the later non-linear.

\subsubsection{Log-Linear Transformation Model (LLTM)}

This model is represented by $\ln D(r)=\alpha+\lambda \ln r w h e r e \quad D(r)=$ Estimated population density of a valley at a distance $r r=$ distance square kilometers $\alpha=$ Constant

\subsubsection{Log-Exponential Transformation Model(LETM)}

LETM is inherently linear model. Inherently linear models are nonlinear but we can be transformed into linear models through a variety of methods. $D(r)=e^{\alpha+\lambda}$ where $D(r)=$ Estimated population density of valley at adistance $r$ from the pivot of valley $r=$ Distance square kilometers $\alpha=$ Constant

$\lambda=$ Distance gradient coefficient parameter.

We will linearize the model by taking the $\operatorname{logs}$ to $\operatorname{get} \ln D(r)=\alpha+\lambda r$.

\section{Result and Discussion}

A comparison of the GFGDM developed by DGT and GFGDM developed by RGT shows that in the formercase as the distance increases the gradient decreases more rapidly and the density curves become more and more flatconfirming the convergence of the original non-linear model to the linear one. Thus GFGDM developed withthe help of RGT is better than the GFGDM developed with the help of DGT. We can further conclude that thegradients will become more flattened if the city size increases. This means that the associated density curvesgo on flattening more and more to finally join in a single straight line.

\section{References}

1. David A. Plane, Peter A. Rogerson. 2001. "TheGeographical Analysis of Population with Applications toPlanning and Business”, John Wiley \& Sons, Inc. Fotheringham AS. 1993. Spatial-interaction models: The Theory of Competing destinations, Environment and planning A-18:19-26.

2. Mills ES. and Tan JP. 1995. "A Comparison of UrbanPopulation Density functions in developed and developing Countries”, Urban Studies. 7: 313-321.

3. Wang F. and Guldmann JM. 1996. "Simulating UrbanPopulation Density with a GravityBased Model”, Socioecon. Sci. 30(4): 245-256, Elsevier Science Ltd.A.S. Monin, GidroMeteoizdat: Leningard, Russia (1982).

4. D. Briggs et al., 2nd Edition, Routledge, London (1997).

5. M.A.K. Yousufzai, M.A. Hussain and M.R.K.Ansari, "Proceedings of Fourth InternationalConference on Recent Advances in SpaceTechnologies", June 11-13, Istanbul, Turkey(2009), ISBN-978-1-4241-3626-2.

6. M. Martin, “International Strategy for DisasterReduction (UN/ISDR)”, United Nations (20072008).

7. M.A. Hussain and M.R.K. Ansari, Arabian Journal for Science and Engg. 32, No. 32A(2007) 189. 